\title{
DEMÊNCIA NA POPULAÇÃO DE PACIENTES DO HOSPITAL DAS CLÍNICAS DA UNICAMP
}

\author{
David W. Silva', Benito P. Damasceno²
}

\begin{abstract}
RESUMO - Objetivo: Descrever subtipos de demência em pacientes atendidos no HC-UNICAMP. M etodo: No período 1989-1998, foram admitidos 261 pacientes com demência (89,7\% estudados retrospectivamente, e $10,3 \%$ prospectivamente); idade 63,5 anos ( $\pm 13,2$ ) e educação 3,6 anos ( $\pm 3,9 ; 25 \%$ analfabetos). 0 diagnóstico de demência e seus subtipos baseou-se no DSM-IV, CAMDEX, NINCDS-ADRDA, NINDS-AIREN/ADDTC, LundManchester, tap-teste liquórico, exames laboratoriais e de neuroimagem. Resultados: Sessenta e cinco pacientes (24,9\%) tinham demência vascular, $62(23,7 \%)$ Alzheimer, $28(10,7 \%)$ hidrocefálica, $29(11,1 \%)$ pseudodemência depressiva, 12 (4,6\%) pós-traumática, 9 (3,4\%) frontotemporal, 14 (5,4\%) demência mista e 38 (14,5\%) causas diversas ou desconhecidas. A demência foi mínima ou leve em 123 pacientes $(47,1 \%)$, moderada em 45 (36,4\%) e grave em 43 (16,5\%). Conclusão: A proporção de demência vascular foi $24,9 \%$, Alzheimer $23,7 \%$, e hidrocefalia de pressão normal $10,7 \%$, contrastando com a da literatura $(20-30 \%, 50 \%$ e $1-4 \%$, respectivamente), provavelmente por se tratar de casos admitidos num hospital terciário. Estudos epidemiológicos são necessários para avaliar a real proporção das síndromes demenciais na população.
\end{abstract}

PALAVRAS-CHAVE: demência, doença de Alzheimer, demência vascular, CAMDEX

\section{Dementia in patients of UNICAMP University Hospital}

ABSTRACT - Objective: To report the relative frequencies of the causes of dementia in patients seen at UNICAMP Hospital. Method: From 1989 to 1998, 261 dementia patients were admitted (89.7\% studied retrospectively, and $10.3 \%$, prospectively); mean age 63.5 years $( \pm 13.2)$, and education 3.6 years $( \pm 3.9 ; 25 \%$ illiterate). Diagnosis of dementia and its subtypes was based on DSM-IV criteria, CAMDEX, NINCDS-ADRDA, NINDS AIREN/ADDTC, Lund-Manchester, besides tap-test, including laboratory and neuroimaging examinations. Results: Sixty five patients (24.9\%) had vascular dementia, 62 (23.7\%) Alzheimer's, 29 (11.1\%) depressive pseudodementia, 28 (10.9\%) normal pressure hydrocephalus, 14(5.4\%) mixed, $12(4.6 \%)$ post-traumatic, 9 (3.4\%) frontotemporal and $38(14.5 \%)$ had diverse or unknown causes. Dementia was minimal or mild in 123 $(47.1 \%)$, moderate in $95(36.4 \%)$, and severe in $43(16.5 \%)$. Conclusion: Frequency of vascular dementia was $24.9 \%$, Alzheimer's $23.7 \%$, and normal pressure hydrocephalus $10.7 \%$, in disagreement with that found in the neurological literature $(20-30 \%, 50 \%$, and $1-4 \%$, respectively), probably because we are dealing with cases admitted to a tertiary university hospital. Epidemiological studies are needed to disclose the true proportion of these dementia syndromes in the population.

KEY WORDS: dementia, Alzheimer's disease, vascular dementia, CAMDEX.

O progresso da medicina e a melhoria da assistência à saúde têm levado a uma maior expectativa de vida e aumento crescente da faixa de idosos da população brasileira, a qual em 2025 passará ao sexto lugar no mundo quanto ao número absoluto de indivíduos com mais de 60 anos. Com o aumento do número de idosos, tornam-se mais frequentes doenças típicas dessa faixa etária, entre elas as demências degenerativas e vasculares, com todas as suas implicações sócio-econômicas ${ }^{1,2}$.

São poucos os estudos brasileiros que avaliam os subtipos etiológicos de demência ${ }^{3-6}$, e apenas um - o de Herrera et al. ${ }^{3}$ - usou metodologia epidemiológica (Tabela 1). Nosso objetivo é descrever as formas de demência encontradas na população de pacientes que procuraram o Hospital das Clínicas (HC) da Universidade Estadual de Campinas (UNICAMP) num período de dez anos (1989 a 1998).

\section{MÉTODO}

O estudo realizou-se em duas fases. Na fase retrospectiva, foram analisados os registros dos casos admitidos de janeiro de 1989 a dezembro de 1998. Foi feita inicialmente a pesquisa no banco de dados de diagnósticos do

Unidade de Neuropsicologia e Neurolinguística, Departamento de Neurologia da Faculdade de Ciências Medicas da Universidade Estadual de Campinas (FCM-UNICAMP), Campinas SP, Brasil: ${ }^{1}$ Mestre em Ciências Médicas; ${ }^{2}$ Professor Associado do Departamento de Neurologia.

Recebido 13 Março 2002, recebido na forma final 3 Junho 2002. Aceito 12 Junho 2002.

Dr. David W. Silva - Avenida Papa Pio XII, 33/22 - 13066-710 Campinas SP - Brasil. 
Tabela 1. Etiologia da demência em estudos brasileiros.

\begin{tabular}{|c|c|c|c|c|c|}
\hline Autor / Local & № Casos & DA (\%) & DV (\%) & $\mathrm{DA}+\mathrm{DV}(\%)$ & Outras (\%) \\
\hline Herrera / Catanduva ${ }^{3}$ & 118 & 54,7 & 9,4 & 14,5 & 19,7 \\
\hline Nitrini / USP 4 & 100 & 54 & 20 & - & 26 \\
\hline Godoy / S.J.R.Preto ${ }^{5}$ & 250 & 55,2 & 27,6 & 12,8 & 4,4 \\
\hline Vale / Ribeirão Preto ${ }^{6}$ & 104 & 33,6 & 19,2 & 3,8 & 43,5 \\
\hline UNICAMP (este estudo) & 261 & 23,7 & 24,9 & 1,9 & 49,5 \\
\hline
\end{tabular}

DA, doença de Alzheimer; DV, demência vascular.

HC-UNICAMP e nos registros do Ambulatório de Neurologia e Unidade de Neuropsicologia e Neurolinguística, utilizando-se as palavras-chave: demência, Alzheimer, múltiplos infartos, arteriosclerose e aterosclerose. A seguir, foram consultados os prontuários dos possíveis casos de demência. A fase prospectiva abrangeu os casos novos de demência admitidos no período de dezembro de 1997 a dezembro de 1998. A análise estatística considerou os casos em conjunto.

Todos os pacientes admitidos no período a que se refere o estudo (1989-1998) foram avaliados no Ambulatório de Neurologia e Unidade de Neuropsicologia e Neurolinguística, passando por: (1) uma anamnese detalhada e objetiva (incluindo entrevista com os familiares); (2) exame neurológico (conforme protocolo do Ambulatório); (3) avaliação neuropsicológica e neuropsiquiátrica; (4) exames laboratoriais de sangue e líquor, com testes hematológicos, bioquímicos, bacteriológicos e sorológicos relevantes; (5) eletrencefalografia; e (6) neuroimagem: tomografia computadorizada (ou ressonância magnética) e cintilografia da perfusão sanguínea (SPECT) cerebral.

Os dados demográficos, clínicos e laboratoriais, tanto retrospectivos como prospectivos, foram registrados num protocolo único que permitia avaliar a etiologia dos casos, excluindo-se aqueles que não preenchiam os critérios de síndrome demencial.

O diagnóstico de demência baseou-se no DSM-IV7 ou (antes do advento deste) no CAMDEX ${ }^{8,9}$. Os critérios operacionais do CAMDEX são superponíveis aos do DSM-IV, exigindo porém que a síndrome demencial esteja presente há pelo menos seis meses. Para o diagnóstico foram considerados os dados da anamnese, exame neurológico, avaliação neuropsicológica com a bateria de Luria ${ }^{10}$ e neuropsiquiátrica-cognitiva com a bateria do CAMDEX (CAMCOG), que foi utilizado tanto em sua forma completa $^{8}$ como reduzida ${ }^{11,12}$, ambas incluindo o Mini Exame do Estado Mental ${ }^{13}$.

Para o diagnóstico dos subtipos etiológicos, foram analisados os dados clínicos, laboratoriais e de neuroimagem, de acordo com os critérios: NINCDS-ADRDA ${ }^{14}$ para doença de Alzheimer (DA); Lund-Manchester ${ }^{15}$ para demência frontotemporal (DFT); tap-teste liquórico ${ }^{16,17}$ (avaliação quantitativa da marcha e funções cognitivas, antes e após a reti- rada de 50 a $100 \mathrm{ml}$ de líquor lombar) para hidrocefalia de pressão normal (HPN); CAMDEX para pseudodemência depressiva (PDD); e os critérios ADDTC ${ }^{18}$ e NINDS- AIREN ${ }^{19}$ combinados para o diagnóstico de demência vascular (DV), considerando como necessário para o diagnóstico o comprometimento de memória, mas não a presença de sinais focais lateralizados; e valorizando os acidentes vasculares hemorrágicos.

\section{RESULTADOS}

Avaliamos 286 casos, dos quais 261 preencheram os critérios de demência. Entre os 25 casos excluídos, houve 10 casos de amnésia, 4 casos de neurose ansiosa, 3 casos de depressão, 2 casos de síndromes focais, 2 casos de deficiência mental e um caso de cada um dos seguintes transtornos: esquizofrenia, síndrome pós-concussão cerebral, declínio

Tabela 2. Etiologia da demência.

\begin{tabular}{lcc}
\hline Etiologia da demência & $\begin{array}{c}\text { Número } \\
\text { de casos }\end{array}$ & $\begin{array}{c}\text { Frequência } \\
(\%)\end{array}$ \\
\hline Vascular & 65 & 24,9 \\
Alzheimer & 62 & 23,7 \\
Hidrocefálica* & 31 & 11,9 \\
Pseudodemência depressiva & 29 & 11,1 \\
Demência mista ** & 14 & 5,4 \\
Secundária a TCE & 12 & 4,6 \\
Frontotemporal & 9 & 3,4 \\
Causas diversas ou & 39 & 15 \\
desconhecidas *** & 261 & 100 \\
\hline Total & & \\
\hline
\end{tabular}

* hidrocefalia de pressão normal (28 casos) e hidrocefalia aguda (3); ** vascular-hidrocefálica (6), vascular-Alzheimer (5), vascular-infecciosa (2) e vascular-metabólica (1); *** demência de etiologia não definida (15); alcoólica (6); degenerativas não-Alzheimer [doença de Parkinson (2), paralisia supranuclear progressiva (2), doença de Huntington (1) ]; deficiência de B12 e folato (4); tumores (3); neuroinfecções (4); hipotireoidismo (1); doença de Jakob-Creutzfeldt (1). TCE, trauma crânio-encefálico. 
cognitivo relacionado à idade e distúrbio de marcha e esfíncter sem comprometimento cognitivo.

No diagnóstico de síndrome demencial, utilizouse o DSM-IV em 186 indivíduos (71,3\% dos casos) e o CAMDEX em 75 indivíduos [28,7\% dos casos, sendo 33 (12,6\%) na forma completa da bateria e 42 (16,1\%), na forma reduzida]. Nesta casuística, a maioria era do sexo masculino $(57,5 \%)$ e de raça branca $(88 \%)$. A idade variou de 20 a 87 anos (média $63,5 \pm 13,2$ ) e o nível educacional, de 0 a 15 anos (média 3,6 3 3,9 anos; $25 \%$ eram analfabetos). Os tipos de demência mais frequentes foram a DV [24,9\% dos casos; 49 DV provável (75,3\% dos casos de DV) e 16 DV possível $(24,7 \%)]$, DA [23,7\% dos casos; 34 DA provável (54,8\% dos casos de DA) e 28 DA possível $(45,2 \%)]$, PDD ( $11,1 \%$ dos casos) e HPN (10,7\% dos casos) (Tabela 2). Conforme a Escala de Gravidade do CAMDEX, a demência foi classificada como mínima em 29 casos $(11,1 \%)$, leve em 94 (36\%), moderada em 95 (36,4\%) e grave em 43 (16,5\%).

\section{DISCUSSÃO}

\section{Dos métodos}

Este estudo não foi planejado com metodologia epidemiológica, por se tratar da investigação de prontuários ou coleta direta de dados em um hospital terciário, referência de Campinas e municípios circunvizinhos. O Ambulatório de Neurologia é um serviço de demanda selecionada, que admite pacientes mediante triagem prévia. Os resultados desse tipo de estudo, embora não configurem verdadeiras estimativas de prevalência populacional, podem contribuir para melhorar a assistência à demência em tais serviços.

A coleta retrospectiva e prospectiva dos dados foi facilitada pela existência de um protocolo único de condutas diagnósticas para os casos de demência e síndromes correlatas, o qual é utilizado no Ambulatório há mais de dez anos. Tal protocolo permite verificar os critérios de inclusão e exclusão do NINCDS-ADRDA para doença de Alzheimer. Apenas em uma pequena parcela dos casos (retrospectivos) alguns dados não puderam ser obtidos.

A prevalência de demência e seus subtipos depende dos critérios diagnósticos empregados ${ }^{20,21}$. O uso do CAMDEX em $29 \%$ dos casos pode ter levado à exclusão daqueles com menos de seis meses de evolução, os quais, entretanto, constituem uma porcentagem insignificante, considerando-se que o tempo médio de evolução dos sintomas até a data da avaliação ambulatorial foi de 38 meses (mediana de 24 meses). No diagnóstico de demência vascular, combinamos o NINDS-AIREN e ADDTC: relativamente a seus pontos de discordância, consideramos necessária a presença de amnésia (tal como definida pelo DSMIV e CAMDEX), consideramos a presença de eventos vasculares isquêmicos e/ou hemorrágicos, desde que capazes de explicar o quadro demencial, mesmo na ausência de sinais de lateralização (certas lesões vasculares, dependendo particularmente de sua localização, podem desintegrar as redes neurofuncionais cerebrais produzindo demência, mas não déficits motores ou sensoriais lateralizados). No diagnóstico de DA, adotamos os critérios NINCDS-ADRDA devido ao seu amplo uso internacional e melhores resuItados nas séries de confrontação anátomo-clínica ${ }^{22}$. Casos de doença de Lewy foram considerados como subtipos de DA. O diagnóstico de DFT baseou-se nos critérios de Lund-Manchester, dando-se atenção aos itens com maior poder discriminativo entre DFT e $\mathrm{DA}^{23,24}$ : perda da consciência pessoal, hiperoralidade, comportamento estereotipado e perseverativo, redução progressiva da fala e preservação relativa da orientação espacial.

Os critérios diagnósticos de HPN requeriam a presença de: (1) progressiva alteração da marcha do tipo apráxico ou parapareto-espástico, com deterioração mental (demência), com urgência-incontinência urinária; (2) neuroimagem mostrando hidrocefalia comunicante, com índice ventricular (Evans) maior que 0,30 e sulcos cerebrais apagados ou normais para a idade, sem sinais de infartos ou lesões parenquimatosas clinicamente relevantes; e (3) pressão liquórica lombar média abaixo de $18 \mathrm{~cm}$ de água. Um tap-teste negativo não afastava HPN e seu objetivo era a obtenção de um índice mais seguro de prognóstico pós-operatório ${ }^{16,17}$.

O diagnóstico de PDD baseou-se na presença de depressão evidente associada a dificuldades cognitivas de menor gravidade (geralmente restritas à orientação e memória), as quais eram inconsistentes, ou seja, desapareciam com o tratamento adequado da depressão. Conforme nosso protocolo de condutas, todos os pacientes com queixas depressivas relevantes foram avaliados com o CAMDEX. Optamos pela escala de depressão do CAMDEX devido ao seu valor diagnóstico demonstrado em outros estudos 9,12,20,25; por ser facilmente aplicável, possuindo apenas 14 itens considerados como os mais discriminativos de outras escalas; e por basear-se em informações obtidas tanto do paciente (subjetivas) como de algum informante (objetivas). 


\section{Dos resultados}

Nossos achados concordam com os de outros estudos de demência no que se refere à idade da maioria de nossos pacientes (média 64 anos), mas discorda quanto ao leve predomínio do sexo masculino (58\%). A alta proporção de indivíduos de raça branca (88\%), embora maior que o esperado para a população brasileira, parece refletir tanto a composição étnica da população regional, com grande número de imigrantes de origem caucasiana, como uma procura mais diferenciada ao nosso sistema hospitalar. O elevado índice de analfabetismo (25\%) encontrado nesta população de pacientes está de acordo com relatos prévios de Damasceno ${ }^{26}$ numa amostra de 92 pacientes e 111 sujeitos controles normais atendidos no HC-UNICAMP.

As proporções de demência vascular (25\%), doença de Alzheimer (24\%), HPN (11\%) e outras demências secundárias (40\%) contrastam com as encontradas na literatura $(20-30 \%, 50 \%$, 1-4\% e $10-15 \%$, respectivamente), provavelmente por se tratar de casos admitidos num hospital terciário, muitos deles enviados por neurologistas da região para um diagnóstico mais detalhado e sofisticado. A casuística estudada por Vale et al. ${ }^{6}$, também em um hospital universitário do interior do Estado de São Paulo, é a que mais se assemelha à nossa quanto à proporção dos subtipos de demência (Tabela 1). Por outro lado, constatamos que, à época do atendimento ambulatorial, a maioria de nossos pacientes apresentava demência em fase moderada a grave, com pelo menos um ano de evolução. Esta demora em procurar o serviço especializado está provavelmente relacionada ao alto índice de analfabetismo eà interpretação dos déficits cognitivos como "caduquice" normal dos velhos.

Em conclusão, nossos achados mostram a necessidade de métodos epidemiológicos para estabelecer a verdadeira proporção e gravidade da síndrome demencial em nossa população.

\section{REFERÊNCIAS}

1. Chaimowicz F. Health of the Brazilian elderly population on the eve of the $21^{\text {st }}$ century: current problems, forecasts and alternatives. Rev Saúde Pública 1997;31:184-200.

2. Instituto Brasileiro de Geografia e Estatística. Censo Demográfico Rio de Janeiro, IBGE 1991/ 1996.
3. Herrera E, Nitrini R, Caramelli P, Silveira ASB. Population epidemiological survey of dementia in Catanduva, São Paulo State. Arq Neuropsiquiat 1998;56(Supl 1):184.

4. Nitrini R, Lefèvre BH, Mathias SC, et al. Evaluation of 100 patients with dementia inSão Paulo, Brazil: correlation with socioeconomic status and education. Alzheimer Dis Assoc Disord 1995;9:146-151.

5. Godoy MRP, Tognola WA, Fernandes MRSF, Verona E, Souza HM. Etiological profile of dementia in an ambulatorial population. Arq Neuropsiquiatr 1998; 56(Supl 1):334.

6. Vale FAC, Miranda SC, Oliveira MF. Perfil clinicodemográfico de pacientes com demência atendidos em um ambulatório de neurologia comportamental. Arq Neuropsiquiatr 2000 (Supl 2):12.

7. American Psychiatric Association. Diagnostic and Statistical Manual of MentalDisorders, 4.Ed. Washington: APA, 1994.

8. Roth M, Huppert F, Tym E, Mountioy CQ. CAMDEX: The Cambridge Examination for Mental Disorders in the Elderly. Cambridge: Cambridge Univ Press, 1988.

9. Cristensen A-L. Luria's neuropsychological investigation 2.Ed. Copenhagen: Munksgaard, 1979.

10. Neri $M$, Roth $M$, Mountjoy CQ, et al. Validation of the full and short forms of the CAMDEX interview for diagnosing dementia. Dementia 1994;5:257-265.

12. Neri M, Rubichi S, Devreese LP, Roth M, Cipolli C. Validation of the full and short forms of the CAMDEX interview for diagnosing dementia: evidencefrom a one-year follow-up study. Dement Geriatr Cogn Disord 1998;9:339-346.

13. Bertolucci PHF, Brucki SMD, Campacci SR, Juliano Y. O mini-exame do estado mental em uma população geral: impacto da escolaridade. Arq Neuropsiquiatr 1994;52:1-7.

14. McKhann G, Drachman D, Folstein M, Katzman R, Price D, Stadlan E. Clinical diagnosis of Alzheimer's disease: report of the NINCDS ADRDA Work Group under the auspices of Department of Health and Human Services Task Force of Alzheimer's disease. Neurology 1984;34:939-944.

15. Brun A, Englund B, Gustafson L, et al. Clinical and neuropathological criteria for frontotemporal dementia. J Neurol Neurosurg Psychiatry 1994;57:416-418.

16. Damasceno BP. O valor preditivo da punção liquórica ("tap-teste") na hidrocefalia de pressão normal. Tese de Livre-Docência, Universidade Estadual de Campinas. Campinas, 2000.

17. Damasceno BP, Carelli EF, Honorato DC, FacureJJ. Thepredictivevalue of cerebrospinal fluid tap-test in normal pressure hydrocephalus. Arq Neuropsiquiatr 1997;55:179-185.

18. Chui HC, Victoroff JI, Margolin D, Jagust W, Shanke R, Katsman R. Criteria for diagnosis of ischemic vascular dementia proposed by the State of California Alzheimer's Disease Diagnostic and Treatment Centers. Neurology 1992;42:473-480.

19. Roman GC, Tatemich TK, Erkinjuntti T, et al. Vascular dementia: diagnostic criteria for research studies. Report of the NINDS-AIREN International Workshop. Neurology 1993;43:250-260.

20. Blessed G, Black SE, Butler T, Kay DWK. The diagnosis of dementia in the elderly: a comparison of CAMCOG (thecognitivesection of CAMDEX), the A GECAT Program, DSM-III, the Mini-Mental State Examination and some short rating scales. Br J Psychiatry 1991;159:193-198.

21. Erkinjuntti T, OstbyeT, Steenhuis R, Hachinsky V. Theeffect of different diagnostic criteria on the prevalence of dementia. N Engl J Med 1997;337:1667-1674.

22. Nitrini R. Diagnóstico dedemência: avaliação clínica, neuropsicológica e através da tomografia por emissão de foton único. Tese de Livre docência, Universidade de São Paulo. São Paulo, 1993.

23. Miller $\mathrm{BL}$, Ikonte $\mathrm{C}$, Ponton $\mathrm{M}$, et al. $\mathrm{A}$ study of the Lund-Manchester Research Criteria for Frontotemporal Dementia: clinical and single photon emission CT correlations. Neurology 1997;48:937-942.

24. Neary D, Snowden JS, Gustafson L, et al. Frontotemporal lobar degeneration : a consensus on clinical diagnostic criteria. Neurology 1998; 51:1546-1554.

25. Lindeboom J, Horst RT, Hooyer C, Dynkgreev M, Jonker C. Some psychometric properties of CAMCOG. Psychol Med 1993;23:213-219.

26. Damasceno BP. Time perception as a complex functional system: neuropsychological approach. Intern J Neuroscience 1996;85:237-262. 